\title{
HEALTH INFORMATION: APPLICATIONS AND CHALLENGES IN THE COVID-19 PANDEMIC
}

\author{
Sheree Lloyd', Sue M Walker2, Ani Goswami³ \\ 1. Griffith University, Queensland Australia \\ 2. Queensland University of Technology, Queensland Australia \\ 3. The Salvation Army Australia Territory
}

Correspondence: s.lloyd@griffith.edu.au

\begin{abstract}
Global health systems are under immense pressure with the exponential growth and spread of COVID-19. Public health and health system responses to the pandemic have relied on health information reporting, visualisation, and projections of incidence, morbidity, and mortality. This commentary aims to explore how health information has been used to inform the public, manage risk, understand capacity, prepare the health system and to plan public health strategy. We also aim to share the health information challenges and our insights to inform future debate and strategic investment. This paper will be relevant to health service and health information managers wanting to understand vulnerabilities and focus for future health information initiatives.
\end{abstract}

\section{KEYWORDS}

Health information, visualisation, COVID- 19

\section{INTRODUCTION}

The planning for, and response to, the COVID - 19 pandemic have relied heavily on health data and information. The importance of reporting systems, coordination of data submissions and presentation of data have been implicit parts of successful responses in countries such as Australia, New Zealand, and Taiwan. Early use of data and information to plan health system capacity, aligned with decisive public health responses have resulted in low community transmission and death rates in these countries.

In this commentary we take a broad view of information management and consider its use for decision making, the uptake of digital health solutions (e.g. telehealth) and the role of data in informing the public in large scale emergency events. From our perspective as health information and health service managers, COVID - 19 has highlighted that health information issues have been prominent. Challenges such as how to best present information, what should be presented to aid comparison and monitoring, privacy, the role of social media, information sharing, telehealth, data quality and relevance of data items will be discussed as well as solutions and options for future investment, planning and debate.

\section{CHALLENGES AND SOLUTIONS}

The reliance on data and information to successfully manoeuvre through the pandemic reinforces the need for strong health information systems, data capture, analysis and reporting to support clinical care and the effective management of the health system including the workforce.

COVID- 19 has also reinforced the need for:

- accurate and timely data provision

- $\quad$ precise counting and consistent definitions 
- consideration of access and equity issues i.e. telehealth infrastructure, the elderly, technological literacy

- $\quad$ prediction and modelling techniques to answer questions such as when will COVID-19 end?

\section{DATA AND INFORMATION USE DURING COVID-19}

Since the emergence of COVID-19 in January 2020, countries like Australia and New Zealand have become familiar with the communication of health information in press conferences, newspapers, social media posts, mobile apps, news, and current affairs programs. The public have been exposed to graphs, charts and numerical tables and understand what is meant by concepts such as flattening the curve. One of the ways that data has been presented to aid this familiarisation has been through graphs and visualisations.

Powerful and simple visualisation of tables for consumption by the public has been critical to what has been regarded as a first-rate response in these countries. We have seen new graph and plot types, for example bullet graphs and bubble charts. Heat maps have been used to show hot spots of disease outbreaks, daily summaries of status of the pandemic including new cases and deaths.

\section{DODGY DATA AND COUNTING}

The World Health Organization (WHO) describes eight pillars in their COVID-19 Strategic Preparedness and Response Plan with data usage prominent, including its use for active monitoring and reporting of disease trends (e.g. case fatality ratios) and for the provision of robust and timely epidemiological and scientific data analysis that can be used to continuously inform and support operational decision making and risk management. [1] Public trust and confidence in the accuracy and representativeness of the data presented is vital.

In Britain, problems with reporting of testing data has contributed to a poor result for the population '.... knowing the scale of infection entails understanding the data. Understanding the data entails trusting the data. And right now Britain's data on testing cannot be trusted' [2]. A news article in June 2020 stated that 'here in Britain we don't know how many people have been tested for the disease'. We know that the testing of all suspected cases provides an understanding of the scale of the outbreak. [3]
In the United States, inconsistencies in public reporting of nursing home deaths may have led to significant underestimates of the death toll [4]. Consequently, in late April the United States did not know how many were dying of COVID-19. [4] This was remedied and the US Centre for Disease Control and Prevention requested that nursing homes start reporting the number of confirmed and presumed cases and deaths.

Predictions are important for planning in the complex environment of a pandemic. Modelling by the Doherty Institute was used to inform the Australian response. Early modelling showed that if the pandemic was allowed to progress without mitigation then the capacity of the Australian health system would be exceeded. [5]

In early days when the progress of the pandemic was uncertain, health service managers in Australia used data to inform the preparation of the health system, predicting staffing shortfalls and requirements and hospital capacity and intensive care bed numbers. This data flowed from multiple sources and resulted in innovative and pragmatic potential solutions. Health Information Managers needed to rapidly upskill to be able to present the data in ways that supported its use for these purposes.

Similarly, the United Kingdom in planning for the way out of lockdown analysed a broad data set that has included NHS 111 (a dedicated number for urgent medical problems) and ambulance calls, GP consultation data, surveillance related to hospital admissions, critical care bed occupancy and deaths.[6]

\section{RIGHT DATA, RIGHT TIME, AND QUALITY}

COVID - 19 pandemic has triggered a tsunami of data and generated enormous amounts of data, referred to as 'datanami'.[7,8] Amidst the chaos, data quality has gained traction globally, as political leaders around the world are embracing the importance of data driven decision making for formulating strategies and reopening their countries.

While different types of datasets are available publicly, there are real concerns about the quality of the data underlying some metrics that are being used to compare and monitor our progress in fighting against the disease. For example, the method of data collection and reporting on metrics such as 'new case counts' and 'hospitalisations' in the United States is not consistent from hospital to hospital.[7] This leads to data quality issues with 
inconsistency in the national 'beds and heads' numbers as well as models that are developed based on these numbers. If we want to compare 'apples with apples' we need to understand the sources and context of data collection.

When examining mortality data, according to the Australian Bureau of Statistics (ABS) the completeness of data is important. The ABS state that lags "between the date of death and date of registration means that only 40 $50 \%$ of those registrations are deaths that occurred in the month being reported'. ABS further notes that disease surveillance systems have been used in Australia to capture COVID-19 data to ensure timeliness. [9]

Raw data is widely reported in the media and on websites leading to challenges in interpretation. Reporting on the basis of numbers of infections and deaths, without any context of population size or the use of rates can contribute to misinterpretations or distortion of the size and impact of pandemic and its progress.

Interpreting data is complex due to differences in definitions, interpretations, and the breadth of data collections. We also need to consider what data indicators mean. To reliably understand death rates between Australia and Sweden, countries that have taken radically different approaches to the management of the pandemic, we need to calculate the cause specific mortality rates.[10] Comparisons of this indicator demonstrate that by managing the pandemic in Australia we have saved 10,383 COVID-19 deaths as a result of our strategy. [10]

According to the Grattan Institute, in Australia at the outset of the pandemic there was no nationally coordinated approach to the release of real time data on confirmed cases and deaths. [1 1] In coming out of the pandemic they suggest the continuing inclusion of a 'surveillance strategy for data collection, analyses and reporting on a national scale. Timely and precise reporting would also help health professionals, government officials and decision makers in getting 'pandemic ready'. [11]

\section{VISUALISATION AND HOW IT HAS BEEN USED DURING THE PANDEMIC}

Visualisation tools can be used to present performance data to synthesise large amounts of data and information to show trends over time.
'The focus is on visual displays in which graphical approaches play a central role in communicating information in a meaningful way. Information visualisations are ubiquitous and critically important to understanding several fields today. With the omnipresent access to large amounts of data, computational techniques have become integral to the burgeoning practice of visualizing data'. [12]

Visualisation of data is a powerful information management reporting tool as large amounts of data can be summarised in ways that tell a story. Visualisation also converts raw data in meaningful ways with high visual appeal to enable intuitive interpretations. [13,14]

Data visualisation tools like Tableau, Qliksense and PowerBI are used to help understand patterns, processes and relationships between the different elements of data. [15] These tools visualise the data in a way that makes the data and or information easy to interpret and digest using colour, size and shapes for example bubble charts $[16,17]$ and as shown in Figure 1 below. [18] This interactive bubble chart by Cheung and cited by Creagh and Kamradt-Scott in the Conversation $[18,19]$ can be fully appreciated by following the link below.

\section{FIGURE 1: COVID 9 INTERACTIVE BUBBLE CHART [18]}

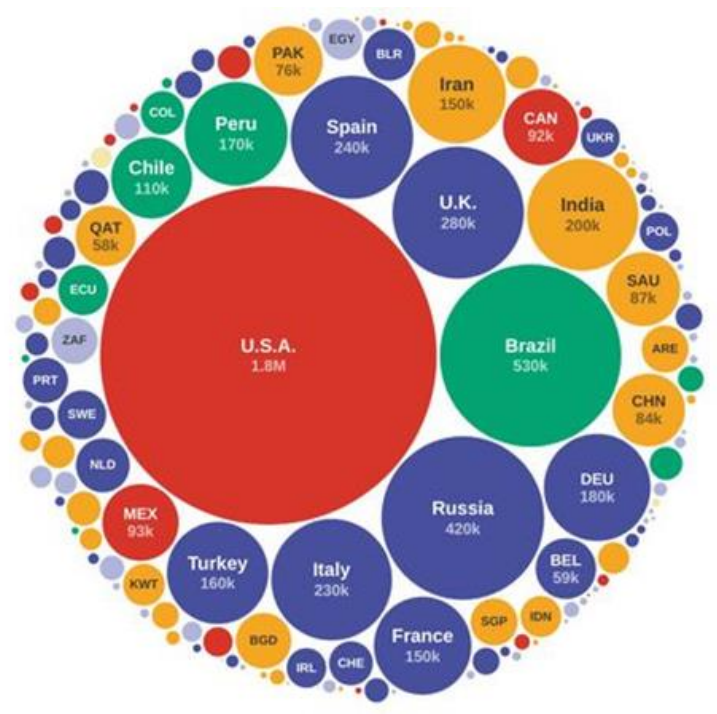

Source: https://observablehq.com/@unkleho/covid-19-bubble-chart-withd3-render

\section{SOCIAL MEDIA}

Social Media influences how we consume news and communications online and past pandemics such as the HINI Influenza pandemic (also known as the Swine Flu), the 
Ebola epidemic and the Zika outbreak all had prominence on social media platforms.

Social media has been used by Federal and State Health Departments in Australia to educate the public and to report daily case numbers as shown in Figure 2 below. [20] Social media has been used during the pandemic to share health information, COVID statistics and promote public health messaging. In New Zealand, Prime Minster Adern regularly uses FaceBook Live to broadcast updates on the COVID- 19 status and response.[21]

FIGURE 2: DAILY FACEBOOK POST SOURCE QUEENSLAND HEALTH [20]

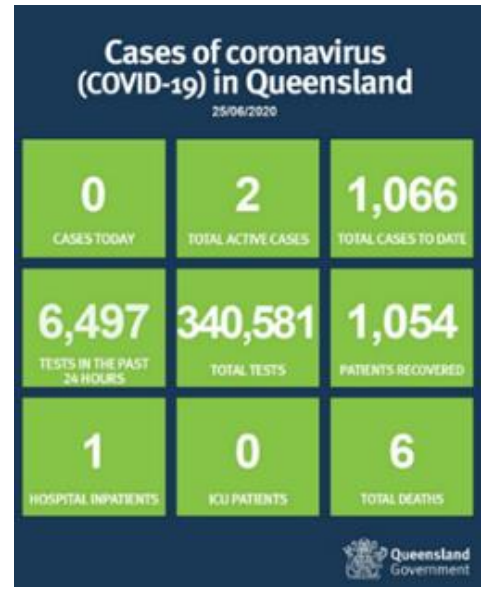

Platforms such as Twitter have been active sharers of information and COVID related research. [22] Controversy and debate have emerged over mask wearing and new evidence reviews have been promoted on Twitter. [23] \#Masks4all.org.uk was established by more than one hundred doctors to promote the wearing of home-made masks to reduce direct transmission and environmental contamination. This group is active on Twitter, Instagram and Facebook and share the emerging evidence on masks.[24]

International and national bodies such as the WHO and the United States Centres for Disease Control and Prevention have expanded their use of social media and websites to communicate important health information to ensure that the population is well informed. The general public have become voracious consumers of health news and information and many are able to discern truth from fiction/fake news. [20]

\section{EHEALTH AND TELEHEALTH}

Past barriers and obstacles slowed the uptake of information and communications technology to support remote patient consultations, but the pandemic has seen an explosion in the use of telehealth. One of the Commonwealth Government's early healthcare interventions was to dramatically expand Australians' access to telehealth.

New 'temporary' items were added to the Medicare Benefits Schedule with speed and include seeking advice from allied health workers and specialists. Changes to facilitate dispensing enabled electronic delivery of prescriptions to the pharmacy, with the ability for patients to have medications delivered to their homes. [25]

Use of telehealth by Australians has been well received, particularly telephone consultations, with more than 4.3 million medical and health services delivered to three million patients in the first month of its availability Minister Hunt quoted in Duckett.[11] A Royal Australian College of General Practitioners (RACGP) survey of more than 1,000 GPs found that 99 per cent of GP practices were now offering telehealth services, with 97 per cent also continuing to offer face-to-face consultations. [11]

\section{PRIVACY}

There have been several privacy related challenges in Australia when it comes to data and the use of technology by the general public: one such example being the COVIDSafe app, a contact tracing mobile application. Whilst uptake of the app was reasonably high this type of application potentially poses threats to an individual's privacy. Concerns related to breaches of the data or unintended uses relating to mass surveillance and data breaches due to insufficient protection.

The Australian Broadcasting Commission, reported by the Sydney Morning Herald noted that epidemiologists and others have been concerned that data collected by the app may be subpoenaed under America's CLOUD Act that can require data storage services, such as Amazon which won the contract to store Australia's data in Australia, to produce personal data irrespective of where it is held. [5]

To address these concerns, in May 2020, the Privacy Amendment (Public Health Contact Information) Act was passed to support the COVIDSafe app and ensure users' privacy is protected. 
In Blackwater, Queensland a major health emergency was called when a resident who had been named, returned a positive COVID-19 result, subsequently and tragically died with an autopsy then confirming other causes of death. This scenario led to community and family fear and distress and was widely reported by the media. It represented a possible breach of the privacy of the family and the individual. [6]

\section{CONCLUSIONS}

The COVID-19 pandemic has exposed health systems' strengths and weaknesses. Data and information have been found to be essential for rapid policy development. In Australia we have been able to manage the outbreak and plan health system capacity and resources because of the rapid availability of relevant data. The data has been used to tell the story of the emerging and continuing pandemic. Not everyone can understand complex concepts, but these can be better explained when visualised. The important role of data scientists, information managers and others in producing the data in easily useable and understandable forms has been vital. However, issues such as privacy and the role of data and information to drive decisions are still prominent. Governance over data and a balance between its use for public good and the maintenance of the privacy of personal information requires strengthening.

Through our analysis we believe that future health information effort and investments should be focussed on ensuring that data for surveillance in pandemics is available when needed, health care organisations have an appropriately skilled workforce equipped with health information, analytics and visualisation skills. We support others who recommend the extension and facilitation of telehealth for effective health care delivery for the future. Processes for the governance of data and information to protect privacy must be robustly agreed after the disruption of the pandemic to protect individuals and society. Social media can be a fast and accurate way to inform and educate the public with wide reach.

The pandemic has demonstrated that those who have strong health information and reporting systems and have used data and information well to prepare for health system readiness and to inform the public have better managed their responses to the pandemic.

\section{COMPETING INTERESTS}

None declared.

\section{References}

1. World Health Organisation. COVID-19 Strategic Preparedness and Response Plan Operational Planning Guidelines to Support Country Preparedness Plan 2020.

2. Conway E. Coronavirus: Dodgy data and double counting - the UK's testing data is a mess 2020.

3. Wood J. The World Health Organization has called on countries to 'test, test, test' for coronavirus - this is why 2020.

4. Peris T. Failure to count COVID-19 nursing home deaths could dramatically skew US numbers. Conversat 2020.

5. Moss R, Wood J, Brown D, Shearer F, Black AJ, Cheng A, et al. Modelling the impact of COVID-19 in Australia to inform transmission reducing measures and health system preparedness. MedRxiv 2020:2020.04.07.20056184. https://doi.org/10.1101/2020.04.07.20056184.

6. Heneghan C, Oke J. UK Data for Assessing COVID-19 Activity 2020.

7. Woodie A (Editor). Datanami News Portal. Datanami 2020.

8. Martineau K. What is the Covid-19 data tsunami telling policymakers? A global team of researchers searches for insights during a weeklong virtual "datathon." n.d.

9. Australian Bureau of Statistics. Provisional Mortality Data: Provisional deaths data for measuring changes in patterns of mortality during the COVID-19 pandemic and recovery period 2020. https://www.abs.gov.au/ausstats/abs@.nsf/mf/3303.0. 55.004 (accessed July 2, 2020).

10. Esterman A. Has Australia really avoided 14,000 coronavirus deaths? Conversat 2020.

https://theconversation.com/has-australia-reallyavoided-14-000-coronavirus-deaths-139465 (accessed July 6, 2020).

11. Duckett S, Mackey W, A S, Swerissen H, Parsonage H. Coming out of COVID-19 lockdown: The next steps for Australian health care 2020

12. Meirelles I. Design for Information: An Introduction to the Histories, Theories and Best Practices Behind 
Effective Information Visualizations, Quatro Publishing Group; 2020, p. 7-16.

13. Brigham TJ. Feast for the Eyes: An Introduction to Data Visualization. Med Ref Serv Q 2016; 35:215-23. https://doi.org/10.1080/02763869.2016.1152146.

14. Ertug G, Gruber M, Nyberg A, Steensma HK. From the Editors-A Brief Primer on Data Visualization Opportunities in Management Research. Acad Manag J 2018; 61:1613-25.

https://doi.org/10.5465/amj.2018.4005.

15. Glesne C. Becoming qualitative researchers: An introduction, 4th edition. 5th ed. Pearson; 2016.

16. [16] Hoelscher J, Mortimer A. Using Tableau to visualize data and drive decision-making. J Account Educ 2018; 44:49-59. https://doi.org/10.1016/j.jaccedu.2018.05.002.

17. Tableau Software Inc. What is Tableau? 2018. https://www.quora.com/What-is-Tableau.

18. Cheung K. COVID-19 Bubble Chart with D3 Render. Observable 2020. COVID-19 Bubble Chart with D3 Render (accessed July 5, 2020).

19. Creagh S, Kamradt-Scott A. Cases, deaths and coronavirus tests: how Australia compares to the rest of the world 2020.

20. Queensland Health. Daily Facebook Feed 2020.

21. Adern J. Facebook \#Live Jacinda Adern 2020.

22. Greenhalgh T. "My peer-reviewed paper is finally out: Face coverings for the public: Laying straw men to rest" - Twitter post 2020.

23. Cochrane UK. Antibody tests have the potential to identify people who've had \#COVID19, but timing makes a big difference to the accuracy of the test n.d.

24. \#Masks4all n.d. https://www.masks4all.org.uk/ (accessed July 5, 2020).

25. Duckett SJ, Stobart A. Australia's COVID-19 response: the four successes and four failures. Publ John Menadue - Pearls Irrit 12 June 2020. https://grattan.edu.au/news/australias-covid-19response-the-four-successes-and-four-failures/. 